NASA Technical Memorandum 105784

$$
\begin{gathered}
11.35 \\
1309 / \\
p .12
\end{gathered}
$$

\title{
2D Velocity and Temperature Measurements in High Speed Flows Based on Spectrally Resolved Rayleigh Scattering
}

Richard G. Seasholtz

Lewis Research Center

Cleveland, Ohio

Prepared for the

Advanced Research Workshop: New Trends in Instrumentation for Hypersonic Research sponsored by NATO

ONERA, Le Fauga-Mauzac, France, April 27-May 1, 1992

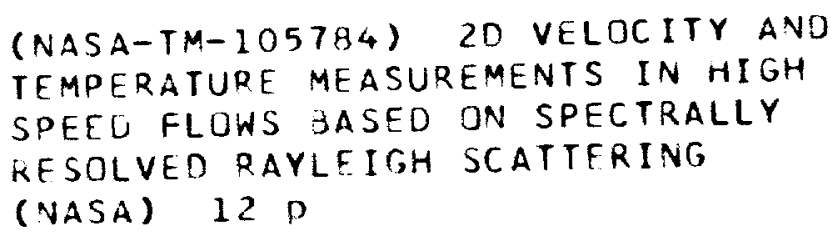




\author{
Richard G. Seasholtz \\ Optical Measurement Systems Branch \\ NASA Lewis Research Center \\ Cleveland, Ohio 44135 \\ U.S.A.
}

\begin{abstract}
The use of molecular Rayleigh scattering for measurements of gas velocity and temperature is evaluated. Molecular scattering avoids problems associated with the seeding required by conventional laser anemometry and particle image velocimetry. The technique considered herein is based on the measurement of the spectrum of the scattered light. Planar imaging of Rayleigh scattering using a laser light sheet is evaluated for conditions at $30 \mathrm{~km}$ altitude (typical hypersonic flow conditions). The Cramer-Rao lower bounds for velocity and temperature measurement uncertainties are calculated for an ideal optical spectrum analyzer and for a planar mirror Fabry-Perot interferometer used in a static, imaging mode. With this technique, a single image of the Rayleigh scattered light from clean flows can be analyzed to obtain temperature and one component of velocity. Experimental results are presented for planar velocity measurements in a Mach 1.3 air jet.
\end{abstract}

\title{
1. Introduction
}

Laser diagnostics based on molecular scattering are receiving increased attention for use in aerospace test facilities. These methods can provide information directly related to the parameters of the molecular state of the gas including temperature, density, and bulk velocity. The elimination of the need for seeding is an important advantage of molecular scattering methods compared to conventional laser anemometry. Of the various molecular scattering techniques, Rayleigh scattering is the simplest and least expensive to implement. Rayleigh scattering has chiefly been used to obtain gas density by measuring the total Rayleigh scattered power. More information, however, is available from the spectrum of the Rayleigh scattered light.

The Rayleigh scattering spectrum is directly related to the molecular velocity distribution function, which is a function of the thermodynamic parameters (temperature, density, pressure), composition, mean velocity, and turbulence parameters. Light scattered at a single scattering angle provides information related to one component of the molecular velocities. Thus one component of the mean velocity can be determined (from the shift of the spectral peak). The width of the spectrum is related to the gas temperature and turbulence. For low turbulence, gas temperature can be obtained; or, for a known temperature, turbulence intensity can be obtained.

Extraction of the parameters of interest (temperature and velocity) from the spectrum can be accomplished using a sharp cutoff atomic or molecular absorption 
filter [Shimizu et al.(1983); Miles et al.(1991)], or with a high resolution spectrometer, such as a Fabry-Perot or Michelson interferometer. Point measurements of Rayleigh scattering spectra with scanning Fabry-Perot interferometers have been used to measure temperature in a hydrogen-air flame [Cattolica et al.(1976); Pitz et al.(1976)] and velocity and temperature in the exhaust of a small hydrogen-oxygen rocket [Seasholtz et al.(1991)]. Rayleigh scattering has been proposed for atmospheric temperature measurements [Schwiesow and Lading(1981); Rees et al.(1984)]. The application of single pass and multi pass scanning Fabry-Perot interferometers for high speed velocity point measurements has been previously analyzed [Seasholtz (1991)]. Imaging velocity measurements of seeded flows using a Michelson interferometer have also been reported by Seiler and Jãger (1983). In this paper we examine an imaging technique to measure velocity and temperature using a planar mirror Fabry-Perot. The technique is an extension of 1D temperatures and number density measurements in a heated air jet [Lock et al.(1992)] and is similar to Fabry-Perot imaging techniques for measurement of instantaneous velocity of solid surfaces [Gidon and Behar (1986)], temperature and velocity in the upper atmosphere [Sivjee et al.(1980)], and solar corona measurements [Chandrasekhar et al.(1981)].

We first analyze a hypothetical planar imaging experiment where the measurement region is illuminated with a laser light sheet, and the Rayleigh scattered light is detected with a 2D array detector. It is assumed that the medium under study is a single component gas with no turbulence and contains no particles. The Cramer-Rao lower bounds for velocity and temperature measurement errors are calculated. We first calculate the error bounds for an ideal spectrum analyzer, which gives a basis with which to compare the performance of spectral measurements made with real instruments. The error bounds are then calculated for a planar mirror Fabry-Perot interferometer used in the static imaging mode. A specific example typical of hypersonic flow environments is discussed.

The use of 2D Rayleigh imaging for velocity measurements is demonstrated with an experiment designed to measure the axial velocity component in a Mach 1.3 supersonic free jet.

\section{Theory}

In this section we obtain theoretical lower bounds for uncertainty in velocity and temperature measurements for two cases where the scattered light is detected with a 2D array detector. The first case assumes that an ideal instrument is used to measure the spectrum. The second case assumes that the spectrum is measured with a Fabry-Perot interferometer operated in a static, imaging mode (this is the technique described in the Experiment section). Only errors resulting from the Poisson statistics of the detected light are considered. Thus these results represent lower bounds for measurement errors.

\subsection{D RAYLEIGH IMAGING DIAGNOSTIC}

Consider a planar imaging experiment where a laser sheet uniformly illuminates a region in the object plane corresponding to the image of an array detector having $\mathbf{N}_{\mathbf{r}}$ rows (measured perpendicular to the beam propagation direction) and $N_{c}$ columns of pixels. Let the detector pixel size be $L_{\mathbf{x}} \times \mathrm{L}_{\mathbf{x}}$ (square pixels) and let the system have a magnification $M$ (i.e., the measurement plane has dimensions $\left.\left(N_{c} L_{x} \times N_{r} L_{x}\right) / M\right)$. 
The expected number of detected photons per pixel can be written [Eckbreth(1988)]

$$
\langle\mathrm{NPP}\rangle=\frac{\mathrm{E}_{1}}{\mathrm{~N}_{\mathrm{r}}} \frac{\mathrm{nL}_{\mathrm{x}} \lambda_{0} \Omega}{\mathrm{hcM}}\left[\frac{\mathrm{d} \sigma}{\mathrm{d} \Omega}\right]
$$

where $E_{1}$ is the laser energy, $\lambda_{0}$ is the laser wavelength, $(d \sigma / d \Omega)$ is the differential Rayleigh scattering cross section, $\mathrm{n}$ is the molecular number density, $\Omega$ is the solid angle of the collected light, $h$ is Planck's constant, $c$ is the velocity of light, and $\epsilon$ is the overall detection efficiency (including detector quantum efficiency and system losses).

As an example, we consider air at $30 \mathrm{~km}$ altitude $(\mathrm{T}=230 \mathrm{~K}, \mathrm{p}=0.0118 \mathrm{~atm}$, $\left.\mathrm{n}=3.83 \times 10^{23} \mathrm{~m}^{-3}\right), \lambda_{0}=532 \mathrm{~nm}, \mathrm{E}_{1}=1 \mathrm{~J}, \mathrm{~L}_{\mathrm{x}}=23 \mu \mathrm{m}, \mathrm{N}_{\mathrm{r}}=384, \mathrm{M} \Omega=0.05(\mathrm{f} / 4$ at $\mathrm{M}=1)$, $\epsilon=0.05$, and $(\mathrm{d} \sigma / \mathrm{d} \Omega)=6.1 \times 10^{-32} \mathrm{~m}^{2} / \mathrm{sr}$. For this case, equation 1 gives $\langle\mathrm{NPP}\rangle=9.4$ detected photons per pixel.

\subsection{SPECTRUM OF RAYLEIGH SCATTERED LIGHT}

For a single component, low density gas with a Maxwellian velocity distribution, the normalized spectrum of the scattered light is [Fabelinskii (1968)]

$$
S(f) d f=\frac{2 \sqrt{\pi}}{a K} e^{-\left[2 \pi\left(f-f_{0}\right)-K \cdot V\right] 2 / a^{2} K^{2}} d f
$$

where $\mathrm{a}=(2 \kappa \mathrm{T} / \mathrm{m})^{1 / 2}(\mathrm{~m}=$ molecular mass; $\kappa=$ Boltzman's constant $)$. The spectral peak is shifted from the laser frequency $f_{0}=c / \lambda_{0}$ by $K \cdot V / 2 \pi$, where $V$ is the mean velocity of the gas, and $K=\mathbf{k}_{\mathrm{s}}-\mathbf{k}_{\mathrm{o}}$ ( $\mathbf{k}_{\mathrm{s}}$ and $\mathbf{k}_{\mathrm{o}}$ being the wave vectors of the scattered and incident light). The magnitude of $\mathbf{K}$ is a function of the scattering angle $\theta_{s}$ and the wavelength $\lambda$

$$
\mathrm{K}=|\mathrm{K}|=(4 \pi / \lambda) \sin \left(\theta_{\mathrm{s}} / 2\right)
$$

The observed Rayleigh scattering spectrum can be expressed as a set of counts

$$
\left\langle n_{j}\right\rangle=G_{R} S\left(f_{j}\right) \Delta f
$$

which are the expected number of counts in frequency interval $f_{j}$ to $f_{j}+\Delta f ; G_{R}$ is the expected total number of counts. The spectrum (eq. 4) is thus a function of a set of three unknown parameters that can be expressed as the vector

$$
\boldsymbol{\alpha}=\left[\mathrm{G}_{\mathrm{R}}, \mathrm{V}_{\mathrm{K}}, \mathrm{T}\right]
$$

where $V_{K}$ is the velocity component along $K$ (i.e., $V_{K}=K \cdot V / K$ ).

\subsection{CRAMER-RAO LOWER BOUND}

The Cramer-Rao lower bound [Whalen(1971)] for the variance of the estimate of the parameter $\alpha_{\mathrm{i}}$ is given by

$$
V\left(\hat{\alpha}_{\mathrm{i}}\right)=\left[\Gamma^{-1}\right]_{\mathrm{i} i}
$$

where $\Gamma$ is the Fisher information matrix given (for Poisson statistics) by [Lading and Jensen(1980)]

$$
\Gamma_{\mathrm{ij}}=\sum_{q} \frac{1}{\left\langle\mathrm{n}_{\mathrm{q}}\right\rangle} \frac{\partial\left\langle\mathrm{n}_{\mathrm{q}}\right\rangle}{\partial \alpha_{\mathrm{i}}} \frac{\partial\left\langle\mathrm{n}_{\mathrm{q}}\right\rangle}{\partial \alpha_{\mathrm{j}}}
$$


If the parameters are uncorrelated, the estimate of the variance of $\alpha_{\mathrm{i}}$ is simply the inverse of the $i^{\text {th }}$ diagonal element of the Fisher matrix elements given by

$$
V\left(\hat{\alpha}_{\mathrm{i}}\right)=\frac{1}{\Gamma_{\mathrm{i} i}}
$$

2.3.1. Ideal Spectrum Analyzer. If we assume that the parameters are uncorrelated, we can easily evaluate the Fisher matrix elements and use equation 8 to get the Cramer-Rao bounds. Expressed as standard deviations $\sigma\left(\alpha_{\mathrm{i}}\right)=\left[V\left(\alpha_{\mathrm{i}}\right)\right]^{1 / 2}$, the lower bounds for measurement uncertainties for velocity and temperature are

$$
\sigma\left(\mathrm{V}_{\mathrm{K}}\right)=\frac{\mathrm{a}}{\left(2 \mathrm{G}_{\mathrm{R}}\right)^{1 / 2}}, \quad \sigma(\mathrm{T})=\frac{2 \mathrm{~T}}{\left(2 \mathrm{G}_{\mathrm{R}}\right)^{1 / 2}}
$$

We now consider a subregion where it is assumed the parameters do not vary. The total number of photoelectron counts due to Rayleigh scattering detected from this subregion is then $G_{R}=\langle N P P\rangle \times$ number of pixels. The size of the subregion determines the spatial resolution of the measurements For example, assuming a $20 \times 20$ array of pixels (which corresponds to $0.5 \mathrm{~mm} \times 0.5 \mathrm{~mm}$ ) and $\langle\mathrm{NPP}\rangle=9.4$ (as given in the above example), we have $G_{R}=3760$ counts, $T=230 \mathrm{~K}$, and $a=370 \mathrm{~m} / \mathrm{s}$. Thus the uncertainties in velocity and temperature are $\sigma\left(V_{k}\right)=4 \mathrm{~m} / \mathrm{s}$ and $\sigma(T)=5 \mathrm{~K}$. Note that the velocity uncertainty is proportional to the square root of temperature.

2.3.2. Fabry-Perot Interferometer. The above error estimates were based on the use of an ideal instrument to measure the spectrum. In this section we perform the error estimation based on measurement of the intensity distribution of light imaged through a planar mirror Fabry-Perot interferometer. The expected number of detected photoelectrons $\langle$ NDP $\rangle$ for the $\mathrm{q}_{\text {th }}$ pixel is

$$
\left\langle\mathrm{NDP}_{\mathrm{q}}\right\rangle=\left\langle\mathrm{NPP}_{\mathrm{q}}\right\rangle \iint \mathrm{S}(\mathrm{f}) \mathrm{I}_{\mathrm{t}}\left(\mathrm{f}, \theta_{\mathrm{r}}\right) \mathrm{df} \mathrm{d} \mathrm{A}_{\mathrm{pixel}}
$$

where the Fabry-Perot transmission function [Vaughan(1989)] is

$$
I_{t}(\psi)=\frac{1}{1+F \sin ^{2}(\psi / 2)}
$$

with $\psi$ being the phase delay of the light for one pass through the interferometer given by

$$
\psi=\frac{4 \pi \mu \mathrm{df} \cos \theta_{\mathrm{r}}}{\mathrm{c}}
$$

and $F=\left(2 N_{R} / \pi\right)^{2}$, where $N_{R}$ is the reflective finesse. In this equation, $f$ is the frequency of the light, $\mu$ is the refractive index of the medium in the Fabry-Perot cavity (taken as unity here), $\mathrm{d}$ is the Fabry-Perot mirror spacing, and $\theta_{\mathrm{r}}$ is the angle between the ray and the optic axis. For simplicity, we neglected spectral broadening due to the finite size of the aperture of the collection optics.

We now select, for this example, a particular Fabry-Perot configuration (mirror spacing $d=15 \mathrm{~mm}$; finesse $N_{R}=20$ ) and numerically evaluate the Fisher matrix elements

$$
\Gamma_{i j}=\sum_{q} \frac{1}{\left\langle N D P_{q}\right\rangle} \frac{\partial\left\langle N P_{a}\right\rangle}{\partial \alpha_{i}} \frac{\partial\left\langle N P_{a}\right\rangle}{\partial \alpha_{j}}
$$


TABLE 1. Lower bounds for uncertainty in velocity and temperature measurements.

\begin{tabular}{llcl}
\hline $\begin{array}{l}\text { Fringe } \\
\text { number }\end{array}$ & $\begin{array}{l}\text { Fringe } \\
\text { radius }\end{array}$ & $\begin{array}{l}\sigma\left(\mathrm{V}_{\mathrm{K}}\right), \\
\mathrm{m} / \mathrm{s}\end{array}$ & $\begin{array}{l}\sigma(\mathrm{T}), \\
\mathrm{K}\end{array}$ \\
\hline 1 & 38 pixels & 14 & 44 \\
2 & 69 & 19 & 49 \\
3 & 90 & 22 & 61 \\
4 & 106 & 24 & 71 \\
5 & 121 & 25 & 68 \\
11 & 185 & 20 & 81 \\
19 & 286 & 24 & 83 \\
& & 4 & 5 \\
\hline
\end{tabular}

where the sum is over the same $20 \times 20$ pixel subregion used above. The results of this calculation are given in Table 1 for several locations of the subregion (denoted by fringe radii). The velocity uncertainty is about $20 \mathrm{~m} / \mathrm{s}$ ( 5 times that achievable with ideal spectrum analyzer) and the temperature uncertainty is about $40-80 \mathrm{~K}$ (10 times that of ideal). In practice, the assumption of constant finesse will not be valid; vignetting reduces the effective number of reflections in the interferometer cavity, thereby decreasing the finesse at larger fringe radii. This means that the finesse must be measured, as is done in the experiment described later in this paper.

\subsection{OTHER CONSIDERATIONS}

The example presented above considered an idealized measurement situation. There are always other factors that degrade measurement accuracy. For instance, if the gas is made up of an unknown mixture of component gases, the spectrum cannot be uniquely related to the temperature. If the flow is highly turbulent, and the measurement is obtained over a duration much greater than the turbulence time scale, the spectrum is broadened with both temperature and turbulence contributing to the spectral width. Thus, if one of these is known, the other can be determined from the spectral width, but both cannot be simultaneously determined. The Gaussian spectrum used in the example occurs only when the molecular mean-free-path is greater than the wavelength $\Lambda=2 \pi / \mathrm{K}$ involved in the scattering process (i.e., $y \equiv \Lambda / \mathrm{mfp}<<1$ ). For values of $\mathrm{y}>1$, collective effects in the molecular velocities become important and must be taken into account in evaluating the spectrum shape. For $\mathrm{y}>>1$, relatively simple continuum models can be used [Clark (1975)]; the spectrum in this case splits into three peaks and is referred to as Brillowin scattering. For the transition region, $y \simeq 1$, the spectral calculations are more involved and kinetic theory models, such as the Tenti S6 model [Tenti et al.(1974)] must be used. In general, ambient conditions fall into the transition region of $y \simeq 1$, while the lower densities typical of hypersonic flows have $y<<1$.

Particles in the flow will strongly scatter. In some cases where the particle number density is low, the images can be processed to remove the obvious particles. If the particle number density is so large that the particles images overlap and 


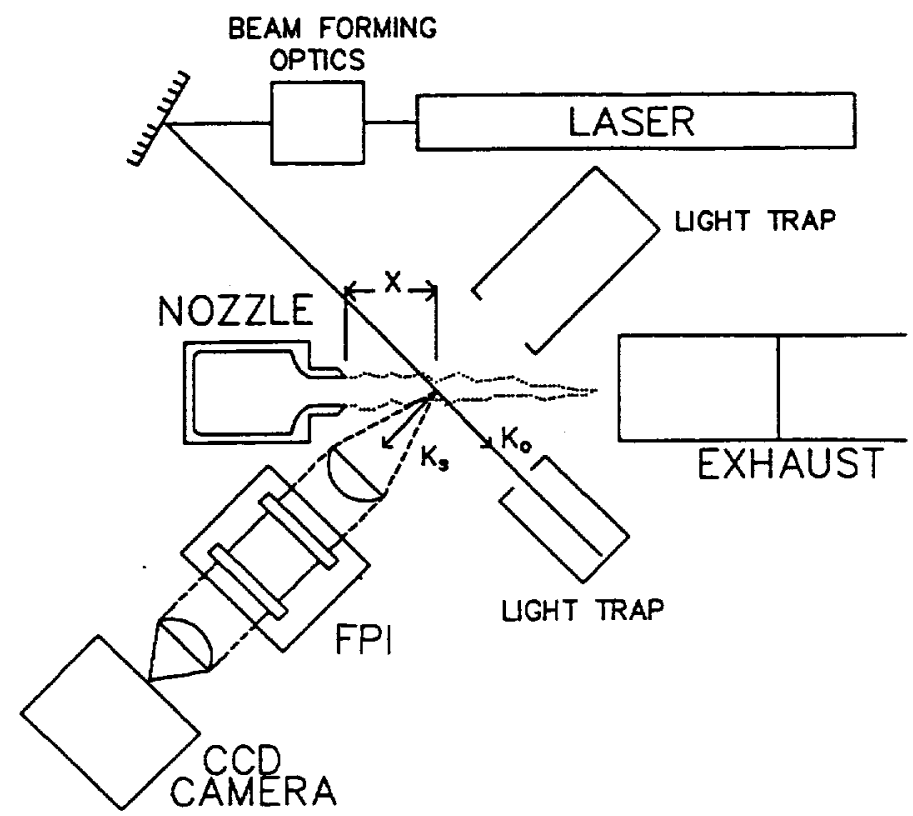

Figure 1. Experimental setup for measurement of velocity in Mach 1.3 free jet using imaging Fabry-Perot interferometer.

cannot be removed, the spectral shape is changed from that of pure molecular scattering and temperature measurements cannot be obtained. It is important to note, however, that particles do not prevent the measurement of velocity (provided there is negligible particle lag) since the spectral peak due to particles is at the same frequency as the peak of the molecularly scattered light.

\section{Experiment to Map Velocity in Supersonic Free Jet}

\subsection{EXPERIMENTAL SETUP}

To demonstrate the use of Rayleigh scattering for measuring velocity, we conducted the experiment shown in figure 1 . The Mach 1.3 supersonic free jet $(9.9 \mathrm{~mm}$ exit diameter) was mounted on a linear translation stage so it could be moved along its axis. The beam from an argon-ion laser $(2.5 \mathrm{~W}, 488 \mathrm{~nm})$ was formed into a sheet (about $10 \mathrm{~mm}$ high by $0.2 \mathrm{~mm}$ thick) using a combination of cylindrical and spherical lenses. The light sheet crossed the flow at $45^{\circ}$. The receiving optics were aligned at $45^{\circ}$ to the flow direction and $90^{\circ}$ to the propagation direction of the laser beam. Thus the $\mathbf{K}$ vector was aligned with the flow, and the axial velocity component was measured. A $250 \mathrm{~mm}$ focal length $\mathrm{f} / 3.6$ lens was used to collect and collimate the scattered light. The collimated light was passed through a planar mirror Fabry-Perot interferometer (70 $\mathrm{mm}$ diameter aperture and $14.96 \mathrm{~mm}$ mirror spacing) and focused onto the CCD array with a $225 \mathrm{~mm}$ focal length lens. The CCD array had 384 rows by 576 columns of $23 \mu \mathrm{m} \times 23 \mu \mathrm{m}$ square pixels (giving a field size in the flow of $9.8 \mathrm{~mm} \times 14.7 \mathrm{~mm}$ ) and was cooled to about $-50^{\circ} \mathrm{C}$ to reduce dark current. The CCD pixel data was digitized with 12 bit resolution and transferred to an 80386 $P C$ via an IEEE-488 interface for storage and analysis.

The initial step in the measurement process was to measure the instrument function $\left[I_{t}(\psi)\right.$, for $\left.f=f_{0}\right]$. A diffusely scattering target was placed in the field of view and illuminated with laser light scattered from a rotating diffuse target (the 


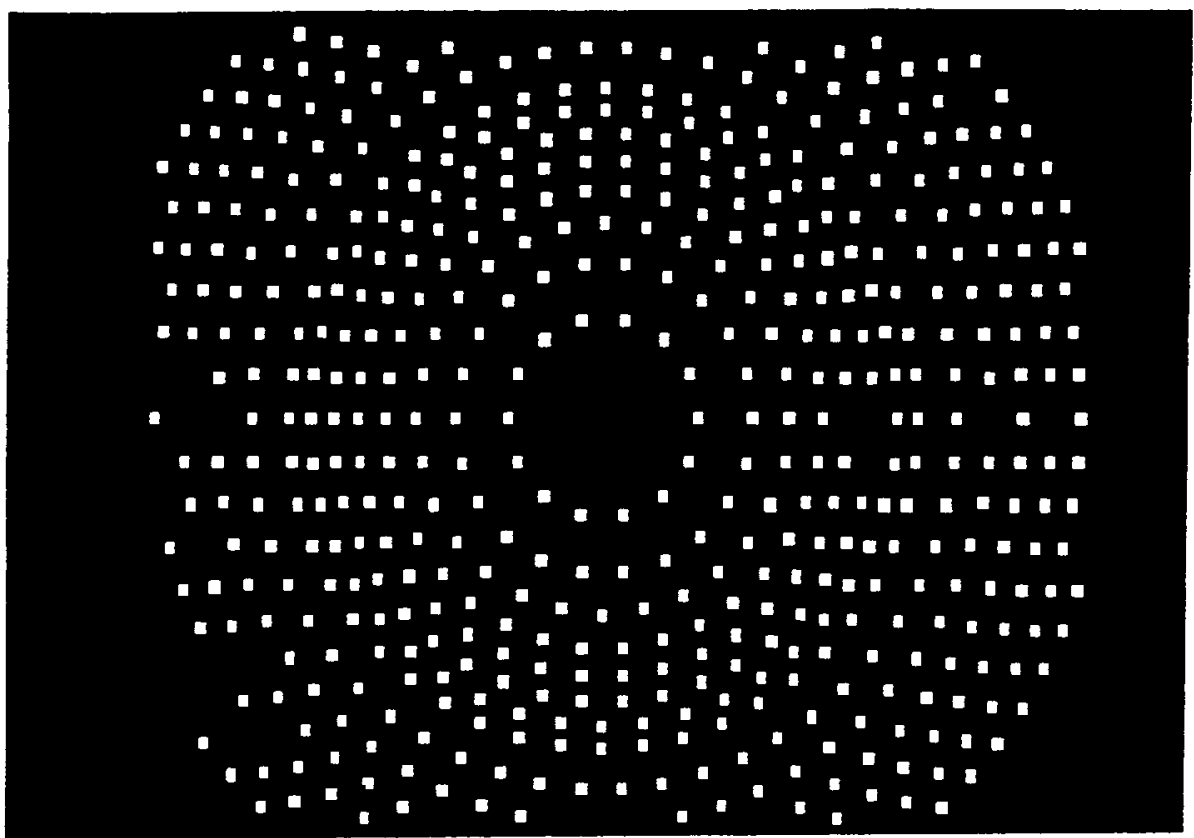

Figure 2. Pattern of subregions used for data analysis $(5 \times 5$ pixel subregions shown; actual size of regions used was $20 \times 20$ pixels).

rotating target was used to reduce speckle in the recorded image. The Rayleigh scattered light from the flow was then recorded. Exposure times were $0.1 \mathrm{~s}$ for the instrument function and 1.0s for the Rayleigh scattering.

Although the air supplied to the nozzle was filtered, it was not feasible to supply filtered air to the air surrounding the jet. Particles in the ambient air were thus mixed into the flow, and temperature measurements were not realizable (even. though the technique described by Kay and Sadler (1991) was used to reduce the effect of particles).

\subsection{DATA REDUCTION}

The data reduction was done in two steps. First, the instrument function image was analyzed to determine the center of the circular fringe pattern, the finesse, and the phase corresponding to the laser frequency. Because the effective finesse and phase varied over the image plane, it was necessary to determine them as a function of location in the image (typically, the finesse varied from about 20 at the center to about 4 at the edges of the image). About $50020 \times 20$ pixel subregions were used. An integral number were positioned around the each of the inner fringes and around every other outer fringe (so the subregions did not overlap). A typical pattern of subregions is shown in figure 2. A $4^{\mathrm{th}}$ order, 2D polynomial (15 parameters) was then fit to these values of phase and finesse. The coefficients for the polynomial fits were saved for the second step (analyzing the image of the Rayleigh scattered light).

In the second step, the image of the Rayleigh scattered light in each subregion was fit to a model function given by equation 10 using the $S 6$ model of Tenti et al.(1974) with the gas properties of air. The velocity data obtained at each subregion were fit to a $2 \mathrm{D} 4^{\text {th }}$ order polynomial, which was used to generate contour plots of constant axial velocity. 


\subsection{RESULTS}

Figure 3 shows constant axial velocity contours for 5 axial locations along the jet axis $(X=20,30,50,70$, and $90 \mathrm{~mm})$. Recall that these planes are oriented at $45^{\circ}$ to the jet axis. At the locations closer to the nozzle exit, the maximum velocity is in good agreement with the $423 \mathrm{~m} / \mathrm{s}$ velocity calculated from the measured total pressure and temperature (assuming isentropic flow). As the measurement plane moves downstream, the maximum velocity decreases as the core flow mixes with ambient air. The contours are elliptically shaped because of the orientation of the measurement plane. We also can see that the center of the jet is shifting to the right as it moves downstream. This is probably caused by a slight misalignment of the jet axis.

The uncertainty in the velocity estimates due to photon arrival rate statistics is quite small, less than $10 \mathrm{~m} / \mathrm{s}$. Errors due other sources most certainly outweigh these statistical errors. One potential error source is the drift in the Fabry-Perot mirror spacing between recording the instrument function and the Rayleigh data. The r.m.s. deviation of the contours from the individual subregions data was about $10-15 \mathrm{~m} / \mathrm{s}$.

The data reduction requires rather lengthy processing, chiefly because of the numerical integration in equation 10 . The time per iteration for each subregion is about 15 s using a $80386 / 33 \mathrm{MHz} /$ Weitek 3167 . Thus an image with 500 subregions and an average of 5 iterations, requires about $10 \mathrm{hr}$ to process. No attempt was made to optimize the code at this time, so it should be possible to substantially reduce the data reduction time.

\section{Concluding Remarks}

The results of this work show the feasibility of using measurements of the spectrum of molecular Rayleigh scattering as a diagnostic suitable for many high speed flow experiments. The technique is capable, under certain restrictions, of mapping gas density, velocity, and temperature.

The experimental work reported here used a $\mathrm{CW}$ argon-ion laser, which results in time-averaged measurements. Use of a pulsed laser and gated detection would offer several advantages. Measurements based on a image from a single laser pulse would give 2D maps of the gas parameters at an instant of time. The effect of background light, such as flame luminosity, could be greatly reduced. And, particle images, if not too dense, should be easier to identify and remove from the flow field image.

A large number density of particles in the flow generally will prevent temperature and density measurements. It is important to note, however, that velocity can be obtained with or without particles, provided the particles follow the flow. Care should be taken to prevent spurious laser light from reaching the receiving optics. Even though this light can be accounted for in the data reduction, it will degrade the signal-to-noise ratio and measurement accuracy. This problem is particularly severe in internal flows where it is very difficult to eliminate all spuriously scattered light. The technique described by Miles et al. (1991) for blocking light at the laser frequency should be useful for high velocity flows where the frequency of the Rayleigh scattered light is shifted well away from the laser frequency. 

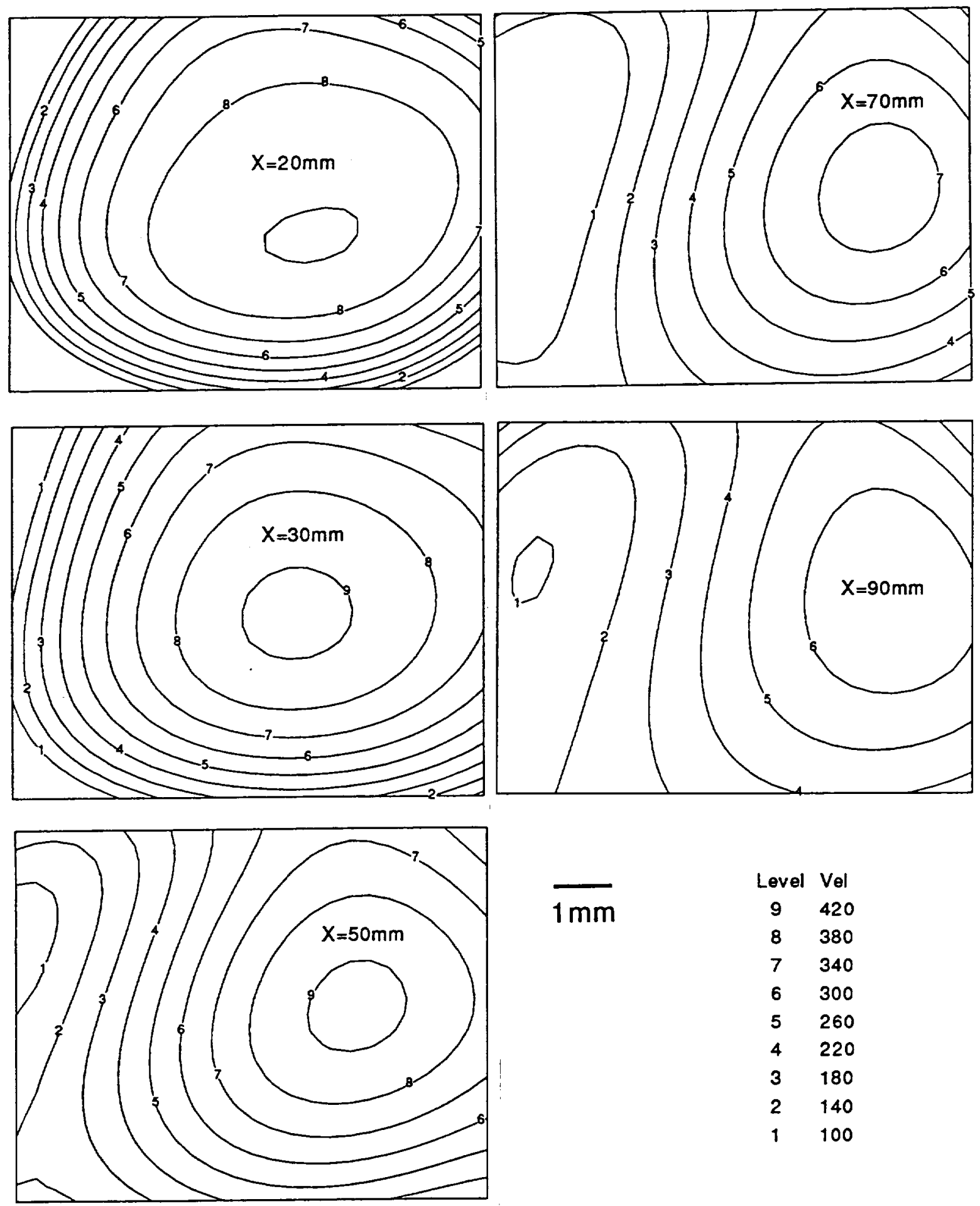

Figure 3. Measured constant axial velocity contours. Distances from nozzle exit are $X=20,30,50,70,90 \mathrm{~mm}$. Isentropic velocity $=422 \mathrm{~m} / \mathrm{s}$. 


\section{REFERENCES}

Cattolica, R., Robben, F. and Talbot, L. (1976) 'The interpretation of the spectral structure of Rayleigh scattered light from combustion gases', AIAA 14th Aerospace Sciences Meeting, Washington, AIAA paper 76-31.

Chandrasekhar, T., Desai, J.N. and Angreji, P.D. (1981) 'Temperatures and differential line-of-sight velocities observed in the solar corona during a total solar eclipse', Appl. Opt. 20, 2172-2173.

Clark, N.A. (1975) 'Inelastic light scattering from density fluctuations in dilute gases. The kinetic-hydrodynamic transition in monoatomic gas', Phys. Rev. A 12, 232-244.

Eckbreth, A.C. (1988) Laser Diagnostics for Combustion, Temperature, and Species, Abacus Press, Tunbridge Wells, Kent, 367.

Fabelinskii, I.L. (1968) Molecular scattering of light, Plenum Press, New York, 263.

Gidon, S. and Behar G. (1986) 'Instantaneous velocity field measurements: application to shock wave studies', Appl. Opt. 25, 1429-1433.

Kay, L. and Sadler, D.A. (1991) 'A method for processing CCD images to remove cosmic rays and other randomly positioned spurious events - theory and experiment', Measurement Science and Technology 2, 532-535.

Lading, L., Jensen, A.S. (1980) 'Estimating the spectral width of a narrowband optical signal', Appl. Opt. 19, 2750-2756.

Lock, J.L., Seasholtz, R.G., and John, W.T. (1992) 'Using Rayleigh-Brillouin scattering to determine one-dimensional temperature and number density profiles of a gas flow field', Appl. Opt. 31, (to be published).

Miles, R.B., Lempert, W.R. and Forkey, J. (1991) 'Instantaneous velocity fields and background suppression by filtered Rayleigh scattering', AIAA 29th Aerospace Sciences Meeting', Reno, AIAA paper 91-0357.

Pitz, R.W. et al.(1976) 'Temperature and density in a hydrogen-air flame from Rayleigh scattering', Comb. and Flame 27, 313-320.

Rees, D. et al.(1984) 'The Doppler imaging system: initial observations of the auroral thermosphere', Planet. Space Sci. 32, 273-285.

Schwiesow, R.L. and Lading, L. (1981) 'Temperature profiling by Rayleigh-scattering lidar', Appl. Opt. 20, 1972-1979.

Seasholtz, R.G., Zupanc, F.J. and Schneider,' S.J. (1991) 'Spectrally Resolved Rayleigh Scattering Diagnostic for Hydrogen-Oxygen Rocket Plume Studies', AIAA 29th Aerospace Sciences Meeting', Reno, AIAA paper 91-0462.

Seasholtz, R.G. (1991) 'High-speed laser anemometry based on spectrally resolved Rayleigh scattering', Fourth International Conference on Laser Anemometry, Cleveland.

Seiler, F. and Jager, W. (1983) 'Flow visualization with Doppler-pictures', Tenth International Congress on Instrumentation in Aerospace Simulation Facilities, Saint Louis.

Shimizu, H., Lee, S.A., and She, C.Y. (1983) 'High spectral resolution lidar system with atomic blocking filters for measuring atmospheric parameters', Appl. Opt. 22, 1373-1381.

Sivjee, G.G., Hallinan, T.J., and Swenson, G.R. (1980) 'Fabry-Perot interferometer imaging system for thermospheric temperature and wind measurements', Appl. Opt. 19, 2206-2209.

Tenti, G., Boley, C.D. and Desai, R.C. (1974) 'On the kinetic model description of Rayleigh-Brillouin scattering from molecular gases', Can. J. Phys. 52, 285-290.

Vaughan, J.M. (1989) The Fabry-Perot Interferometer, History, Theory, Practice and Applications, Adam Hilger, Bristol, Chapter 3.

Whalen, A.D. (1971) Detection of signals in noise, Academic Press, New York 324-331. 


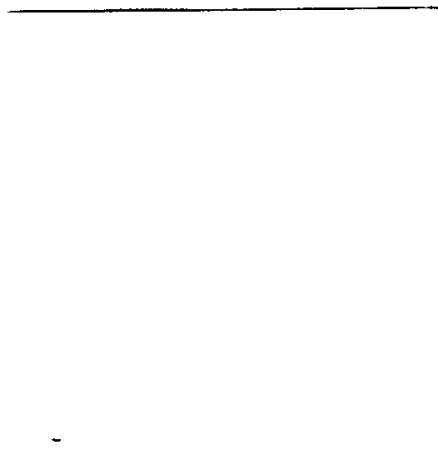

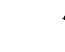


Public reporting burden for this collection of information is estimated to average 1 hour per response, including the time for reviewing instructions, searching existing data sources, gathering and maintaining the data needed, and completing and reviewing the collection of information. Send comments regarding this burden estimate or any other aspect of this

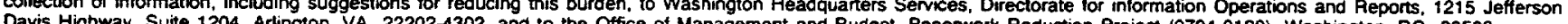
Davis Highway, Suite 1204, Arlington, VA 22202-4302, and to the Office of Management and Budget. Papenwork Reduction Project (0704-0188). Washington. DC 20503.

\begin{tabular}{|l|l|l}
\hline 1. AGENCY USE ONLY (Leave blank) & $\begin{array}{c}\text { 2. REPORT DATE } \\
1992\end{array}$ & $\begin{array}{r}\text { 3. REPORT TYPE AND DATES COVERED } \\
\text { Technical Memorandum }\end{array}$
\end{tabular}

4. TITLE AND SUBTITLE

2D Velocity and Temperature Measurements in High Speed Flows Based on Spectrally Resolved Rayleigh Scattering

\section{AUTHOR(S)}

Richard G. Seasholtz

\section{FUNDING NUMBERS}

WU-505-62-50

\section{PERFORMING ORGANIZATION NAME(S) AND ADDRESS(ES)}

National Aeronautics and Space Administration

Lewis Research Center

Cleveland, Ohio 44135-3191
6. PERForming organization REPORT NUMBER

E-7214

\section{SPONSORING/MONITORING AGENCY NAMES(S) AND ADDRESS(ES)}

National Aeronautics and Space Administration

Washington, D.C. 20546-0001
10. SPONSORINGMONITORING AGENCY REPORT NUMBER

NASA TM-105784

\section{SUPPLEMENTARY NOTES}

Prepared for the Advanced Research Workshop: New Trends in Instrumentation for Hypersonic Research sponsored by NATO, ONERA, Le Fauga-Mauzac, France, April 27-May 1, 1992 . Responsible person, Richard G. Seasholtz, (216) $433-3754$.

12a. DISTRIBUTION/AVAILABILITY STATEMENT

Unclassified - Unlimited

Subject Category 35 12b. DISTRIBUTION CODE

\section{ABSTRACT (Maximum 200 words)}

The use of molecular Rayleigh scattering for measurements of gas velocity and temperature is evaluated. Molecular scattering avoids problems associated with the seeding required by conventional laser anemometry and particle image velocimetry. The technique considered herein is based on the measurement of the spectrum of the scattered light. Planar imaging of Rayleigh scattering using a laser light sheet is evaluated for conditions at $30 \mathrm{~km}$ altitude (typical hypersonic flow conditions). The Cramer-Rao lower bounds for velocity and temperature measurement uncertainties are calculated for an ideal optical spectrum analyzer and for a planar mirror Fabry-Perot interferometer used in a static, imaging mode. With this technique, a single image of the Rayleigh scattered light from clean flows can be analyzed to obtain temperature and one component of velocity. Experimental results are presented for planar velocity measurements in a Mach 1.3 air jet.

\begin{tabular}{|c|c|}
\hline $\begin{array}{c}\text { 14. SUBJECT TERMS } \\
\text { Rayleigh scattering; Fabry-Perot interferometers }\end{array}$ \\
\hline $\begin{array}{c}\text { 17. SECURIT CLASSIFICATION } \\
\text { OF REPORT } \\
\text { Unclassified }\end{array}$ & $\begin{array}{c}\text { 18. SECURITY CLASSIFICATION } \\
\text { OF THIS PAGE } \\
\text { Unclassified }\end{array}$ \\
\hline
\end{tabular}

\begin{tabular}{|c|c|}
\hline & $\begin{array}{c}\text { 15. NUMBER OF PAGES } \\
12\end{array}$ \\
\hline & $\begin{array}{r}\text { 16. PRICE CODE } \\
\mathrm{AO} 3\end{array}$ \\
\hline $\begin{array}{l}\text { 19. SECURTYY CLASSIFICATION } \\
\text { OF ABSTRACT } \\
\text { Unclassified }\end{array}$ & 20. LIMITATION OF ABSTRACT \\
\hline
\end{tabular}


National Aeronautics and Space Administration

Lewis Research Center

Cleveland, Ohio 44135

Othetel Budines:

Penelty for Prtvete Uase $\$ 500$
FOUATH CLASS MAIL.

ADDRESS CORRECTION REQUESTED

National Aeronautics and

Soace Administralion

NASA 45 : 

• 